\title{
Novel LC pseudo switched capacitor filter suited for wireless RF applications
}

\author{
Ahmed El Oualkadi ${ }^{1 a)}$, Jean-Marie Paillot ${ }^{1}$, and Hervé Guegnaud ${ }^{2}$ \\ ${ }^{1}$ Laboratoire d'Automatique et d'Informatique Industrielle, \\ Institut Universitaire de Technologie d'Angoulême, Université de Poitiers, 4, avenue \\ de Varsovie, 16021 Angoulême Cedex, France \\ ${ }^{2}$ EADS-TELECOM \\ Rue Jean-Pierre Timbaud, 78063 Saint Quentin en Yvelines, France \\ a)ahmed.eloualkadi@ieee.org
}

\begin{abstract}
Switched capacitor filters have been in wide-spread used for a few years, for the realization of stable, accurate and high quality filters for operating frequencies below the megahertz range. This paper would like to prove the feasibility of a novel tuned LC pseudo switched capacitor filter with high quality factor of several hundreds for wireless radio frequency applications. The filter is designed with standard $0.35 \mu \mathrm{m}$ silicon BiCMOS technology. Experimental results at $442 \mathrm{MHz}$ switching frequency demonstrate interesting performances; the quality factor is equal to 316 for a frequency bandwidth equals to $1.4 \mathrm{MHz}$.
\end{abstract}

Keywords: switched capacitor, high-Q, RF, analog bandpass filter, EVM

Classification: Integrated circuits

\section{References}

[1] P. J. Chang, A. Rofougaran, and A. A. Abidi, "A CMOS channel-select filter for a direct-conversion wireless receiver," IEEE J. Solid-State Circuits, vol. 32, no. 5, pp. 722-729, May 1997.

[2] A. Yoshizawa and Y. P. Tsividis, "Anti-blocker design techniques for MOSFET-C filters for direct conversion receivers," IEEE J. Solid-State Circuits, vol. 37, no. 3, pp. 357-364, March 2002.

[3] J. C. Nallatamby, M. Bridier, M. Prigent, and J. Obregon, "Switched capacitors and sampled circuit by harmonic balance and related techniques," Electron. Lett., vol. 27, pp. 2364-2367, 1991.

[4] U. Kleine, W. Brockherde, A. Fettweis, B. J. Hosticka, J. Pandel, and G. Zimmer, "An integrated six-path wave-SC filter," IEEE J. Solid-State Circuits, vol. 20, no. 2, pp. 632-640, April 1985.

[5] H. Wupper, "A modified N-path filter suited for practical realization," IEEE Trans. Circuits Syst., vol. 21, pp. 449-456, May 1974.

[6] A. W. Buchwald, K. W. Martin, A. K. Oki, and K. W. Kobayashi, "A 6-GHz integrated phase-locked loop using AlCaAs/Ga/As heterojunction bipolar transistors," IEEE J. Solid-State Circuits, vol. 27, pp. 1752-1762, Dec. 1992.

[7] B. Lai and R. C. Walker, "A monolithic $622 \mathrm{Mb} / \mathrm{s}$ clock extraction data retiming circuit," ISSCC Dig. Tech. Papers, pp. 144-144, Feb. 1993. 
[8] A. El Oualkadi, J. M. Paillot, H. Guegnaud, R. Allam, and L. Dascalescu, "New Command Circuit Design for Tuning High-Q Pseudo 8path Switched-Capacitor Filter," IEICE Electron. Express, vol. 1, no. 13, pp. 363-367, Oct. 2004.

\section{Introduction}

Recent years have seen the rapid proliferation of wireless applications circuits and systems. Wireless transceivers are an increasingly important commercial application $[1,2]$. The current transceiver solutions often use low cost CMOS for digital and baseband circuitry, while reserving silicon bipolar or GaAs for critical radio frequency $(\mathrm{RF})$ circuits. While this is good for optimizing the performance of individual subsystems, it is costly and makes full transceiver integration very difficult. Discrete passive components commonly used in today's transceivers (e.g. SAW filters and high-Q resonators) enable excellent filtering and ease generation of spectral pure signals, but they are often bulky and expensive. In addition, while the fixed frequency characteristics of these components are ideal for single-standard transceivers, they are inflexible and generally not as well suited for systems requiring multi-standard adaptation.

In this context, promising perspectives can be profiled for the switched capacitor filters [3]. These filters present very interesting advantages in particular a very high selectivity associated with the possibility of adjustment of the center frequency by an internal or external clock signal. This characteristic is more significant for the RF integrated circuits, for which the tuning of the center frequency by a clock signal permits either to compensate the dispersions due to the technology used, or to filter various channels with the same filter.

This paper presents the design of a new high-Q LC pseudo switched capacitor filter tunable over a RF broadband for wireless applications.

\section{Architecture of the proposed filter}

Figure 1 shows the architecture of the proposed filter. This novel topology is based on the modification of the basic cell of the well-known scheme of the classical N-path filter $[4,5]$. The new basic cell is formed by a second-order LC low-pass filter. The low value resistor $\mathrm{R}_{\mathrm{L}}$ simulates the inductor losses.

This novel architecture presents the same behavior as the classical N-path filters [5]. Then, this filter can be used for clock recovering by filtering the harmonic components $[6,7]$. It can also be used as bandpass filter centered around Fo. This last application will be discussed hereafter.

A trade-off has been found concerning the number of branches of switched capacitors. Indeed, if a significant number of branches would permit to improve the filter performances, on the other hand it would lead quickly to an excessive complexity of the design [8]. The compromise retained for this study is eight branches; this number seems to be an acceptable limit of the 


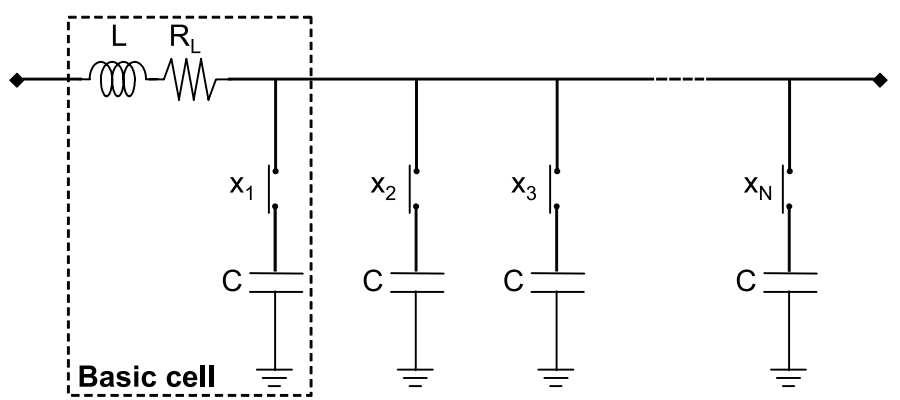

Fig. 1. The proposed filter: LC pseudo switched capacitor filter.

complexity in the realization on-chip.

Let's consider only the basic cell, thus, when the switch $\mathrm{X}_{1}$ is $\mathrm{ON}$, it presents a resistor value equal to $R_{o n}$. In this case, the quality factor of this basic cell (second-order LC low-pass filter) can be written as:

$$
\mathrm{Q}=\frac{1}{\mathrm{R}_{\mathrm{L}}+\mathrm{R}_{\mathrm{on}}} \sqrt{\frac{\mathrm{L}}{\mathrm{C}}}
$$

From (1), the quality factor of the new basic cell depends mainly on the inductor value $\mathrm{L}$. Then, to obtain a high $\mathrm{Q}$-factor it appears evidently that the inductor value should be as high as possible. Therefore, a trade-off must be made between the inductor value and the possibility of its integration.

The main advantages to change the basic cell are to reduce the thermal noise figure due to the input resistor of the classical N-path filter as well as to enhance the quality factor according to input inductor value. Moreover, this new architecture will allow a good dynamic range due to the second order behavior brought by the new basic cell.

\section{Design and simulation results}

The filter switches were produced with $\mathrm{N}$ channel MOSFETs transistors, which are switching sequentially between the ON and OFF modes.

The first critical design limitation is the choice of the switching transistors size. Indeed, a compromise must be found considering the following factors:

- A significant transistors gate size would ensure a low Ron resistor but the associated parasitic capacitor Cds would be significant. Under these conditions, the filter bandwidth would be limited by the cumulated effect of all these parasitic capacities involving attenuation on the whole transfer characteristic, and thus decreasing the filter dynamic range.

- A too small transistors gate size would also involve a reduction of the filter dynamic by the raising of transfer characteristic (floor). Indeed, if Ron value is significant than the compensation of the attenuation is done at low frequencies. Consequently, the frequency which the phenomenon of "the floor" appears is reduced, thus decreasing the dynamic range.

Therefore a compromise about the transistor size gate was found, it consists in using a $\mathrm{N}$ channel MOSFETs of a gate width and length equal to $6 \times 25 \mu \mathrm{m}$ and $0.35 \mu \mathrm{m}$, respectively. 
The command circuit has long been the main obstacle to make a more advanced RF study of this type of filters. The classical solution was generally carried out using a shift register. This solution which required a clock frequency equal to the filter center frequency multiplied by the number of branches to commutate can be exclusively used at low frequencies. An original solution was proposed recently in [8] which consists in using a command circuit made up by a ring voltage controlled oscillator (VCO) with "XOR" gates.

The original association of such command circuit with switched capacitor filters present significant advantages in particular the reduction of command signal frequency (switching frequency) compared to a low frequency solution by a shift register [8]. Moreover, this association can make this type of filters more attractive for designers.

Simulations realized with the novel architecture (tuned LC pseudo switched capacitor filter), show the same transfer characteristic behavior compared with the classical tuned RC switched capacitor filter [8].

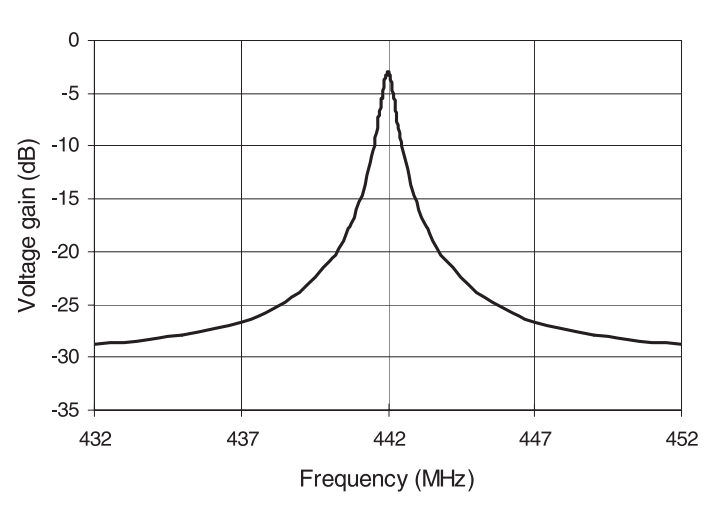

(a)

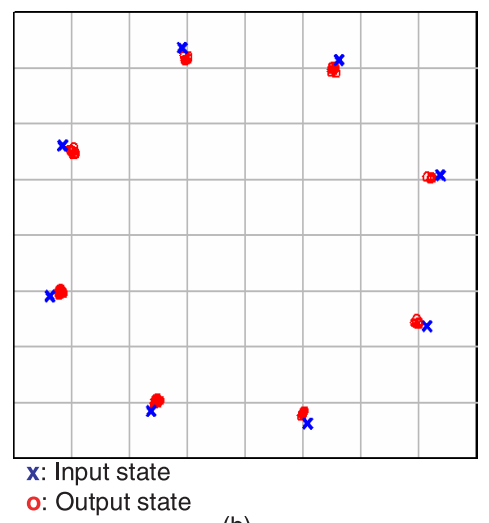

(b)

Fig. 2. (a) Simulated voltage gain versus frequency for $442 \mathrm{MHz}$ switching frequency, (b) constellation diagram at the input and output of tuned LC pseudo switched capacitor filter.

Figure 2 (a) shows the simulated voltage gain versus frequency with a switching frequency Fo equal to $442 \mathrm{MHz}$, for the proposed filter $(\mathrm{L}=20 \mathrm{nH}$, $\mathrm{C}=50 \mathrm{pF}$ ). For these global circuit simulations, all the parasitic effects are taken into account. An optimal dynamic range of $27 \mathrm{~dB}$ is obtained which is comprised between a $-3 \mathrm{~dB}$ voltage gain in the band and a $-30 \mathrm{~dB}$ in the rejection band. This new filter design presents an adjustable simulated center frequency ranging between $325 \mathrm{MHz}$ and $550 \mathrm{MHz}$ with a $900 \mathrm{kHz}$ bandwidth and a simulated quality factor value higher than 360 within the tunable frequency band.

The tuned LC pseudo switched capacitor filter behavior has been simulated when its input signal is changed to a digitally modulating signal of type $\pi / 4-$ DQPSK (Differential Quadriphase Shift Keying) modulation. The choice of such differential modulation is justified by its use in several stan- 
dards of mobile phones.

Figure 2 (b) shows the obtained output constellation compared with the ideal one in case of correlated command sources generated by the ring VCO. These constellations are obtained at a switching frequency equal to $500 \mathrm{MHz}$, using a $\pi / 4-D Q P S K$ digital modulation with a symbol rate equal to $24.3 \mathrm{KHz}$, by applying the same simulated phase noise value on command signals $(-111 \mathrm{dBc} / \mathrm{Hz}$ at $1-\mathrm{MHz}$ of the carrier frequency) [8].

Expressed in percentage (\%), the EVM (Error Vector Magnitude) is a parameter which makes it possible to quantify the effect of the additive noise on the deformation of the transmitted signal constellation.

Table I. Simulated EVM Values.

\begin{tabular}{l|l}
\hline $\begin{array}{l}\text { EVM (RMS) } \\
\text { correlated noise }\end{array}$ & $\begin{array}{l}\text { EVM (RMS) } \\
\text { uncorrelated noise }\end{array}$ \\
\hline $1.314 \%$ & $9.526 \%$ \\
\hline
\end{tabular}

Table I shows that the RMS value of the EVM does not exceed $1.5 \%$ if the noise is correlated. While this value is close to $10 \%$ in the case when the command signals are uncorrelated by using independent switching command sources. From this results, it's appears important to have only one source that provides all commands signals, which confirm our choice of this Ring VCO to command the filter.

\section{Measured results}

A tuned LC pseudo switched capacitor filter was fabricated in standard $0.35 \mu \mathrm{m}$ silicon BiCMOS technology. The values of the inductor (L) and the capacitor (C) are equal to $20 \mathrm{nH}$ and $50 \mathrm{pF}$, respectively. The number of branches retained for this design is eight; this number seems to be an acceptable limit of the complexity of realization on-chip. The corresponding chip layout is shown in Figure 3 (a), and the chip area is $1.1 \times 1.75 \mathrm{~mm}^{2}$. To optimize the filter behavior according the inductance value, this inductor has not been integrated on this first prototype of the chip. The feasibility to integrate the optimized value of the inductance will be studied while developing a prototype in next stage.

The first measured results show a filter center frequency adjustment between $100 \mathrm{MHz}$ and $520 \mathrm{MHz}$. Figure 3 (b) shows the measured voltage gain module versus frequency closed to a switching frequency Fo equal to $442 \mathrm{MHz}$, for tuned LC pseudo switched capacitor filter.

From the Figure 3 (b), the measured dynamic range is roughly $26 \mathrm{~dB}$ and the $-3 \mathrm{~dB}$ bandwidth is about $1.4 \mathrm{MHz}$, thus a quality factor value approximately equal to 316 . By decreasing the command signals magnitudes, the factor quality has been improved (360) but with a reduction in the dynamic range by $5 \mathrm{~dB}$. 


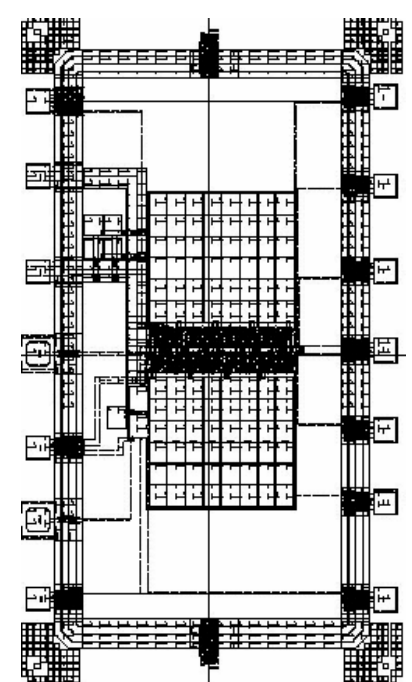

(a)

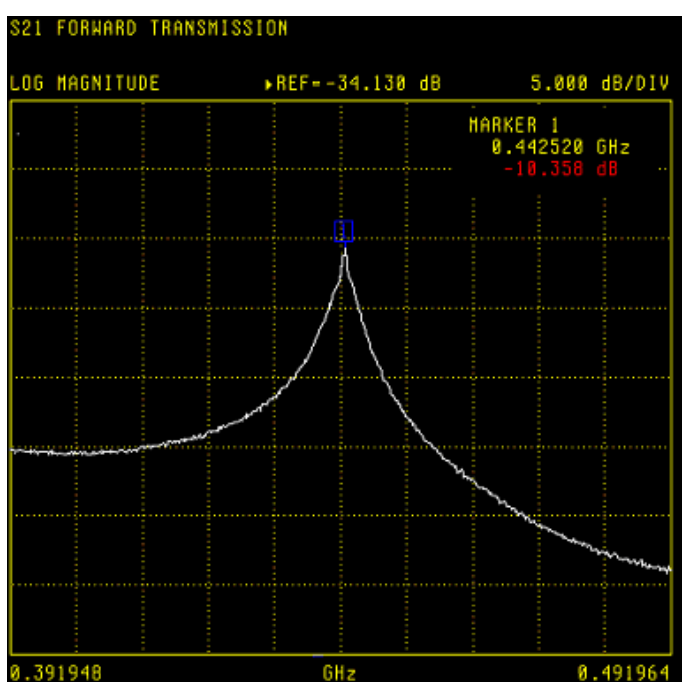

(b)

Fig. 3. (a) Layout plot of the LC pseudo switched capacitor filter and its command circuit, (b) measured S21 parameter for $442 \mathrm{MHz}$ switching frequency.

\section{Conclusion}

A novel architecture represented by a tuned LC pseudo switched capacitor filter has been proposed and studied, this original topology presents a high performance compared to the classic one (N-path filter) particularly in the noise factor and dynamic range. Thereafter, the design of a tuned LC pseudo switched capacitor filter has been discussed, and the first measured results have been presented, these measurements confirm the interest of these original circuits. This circuit could substitute SAW filters in modern wireless RF systems.

\section{Acknowledgments}

This work has been partly funded by a FEDER grant-in-aid from the European Union and the Multi-Mode Modules for Mobile Terminals project in collaboration with ALCATEL Microelectronics foundry. 